\title{
Relaxation and decoherence in a resonantly driven qubit
}

\author{
Zhongyuan Zhou ${ }^{1,2}$, Shih-I Chu ${ }^{1}$, and Siyuan $\mathrm{Han}^{2}$ \\ ${ }^{1}$ Department of Chemistry, University of Kansas, Lawrence, KS 66045 \\ ${ }^{2}$ Department of Physics and Astronomy, University of Kansas, Lawrence, KS 66045
}

\begin{abstract}
Relaxation and decoherence of a qubit coupled to environment and driven by a resonant ac field are investigated by analytically solving Bloch equation of the qubit. It is found that the decoherence of a driven qubit can be decomposed into intrinsic and field-dependent ones. The intrinsic decoherence time equals to the decoherence time of the qubit in free decay while the fielddependent decoherence time is identical with the relaxation time of the qubit in driven oscillation. Analytical expressions of the relaxation and decoherence times are derived and applied to study a microwave-driven SQUID flux qubit. The results are in excellent agreement with those obtained by numerically solving the master equation. The relations between the relaxation and decoherence times of a qubit in free decay and driven oscillation can be used to extract the decoherence and thus dephasing times of the qubit by measuring its population evolution in free decay and resonantly driven oscillation.
\end{abstract}

PACS numbers: 03.67.Lx, 31.70.Hq, 76.60.Es 


\section{INTRODUCTION}

In recent years, significant progress has been made on physical implementation of quantum computation based on superconducting qubits. Quantum coherence has been successfully demonstrated in a variety of superconducting single-qubit systems [1, 2, 3, 4, 5, 6, 7, 8] and coupled two-qubit systems, 9, 10, 11, 12] indicating the potential of superconducting qubits in quantum computing. However, due to unavoidable coupling with environment, the superconducting (charge, flux, and phase) qubits always suffer from decoherence such as relaxation and dephasing, resulting in relatively short coherence times. For this reason, environment-induced decoherence has been and is still a main obstacle to the practical application of superconducting qubits in quantum computation. [2, 13, 14, 15, 16]

The environment-induced decoherence of superconducting qubits has been extensively studied both theoretically 15, 16, 17, 18, 19, 20, 21, 22, 23, 24, 25, 26, 27, 28, 29, 30, 31, 32, 33] and experimentally [2, 8, 13, 21, 25, 34, 35, 36, 37, 38, 39, 40 in the absence of ac driving fields (free decay). Quite a few proposals, such as dynamical decoupling, [41, 42, 43, 44, 45, 46, 47, 48] decoherence free subspaces, [49, 50, 51, 52] spin echoes, [2, 7, 34] and coherencepreserving qubits, [53] have been proposed to reduce such kind of decoherence. However, in superconducting-qubit based quantum computation, ac fields (e.g., microwave fields) are usually used to manipulate the qubit's state. $[1,2$, 3, 4, 5, 6, 7, 8, 9, 10, 11, 12, 18] Recent experiment [14] shows that the decoherence time of a superconducting qubit is significantly increased in the presence of a resonantly ac driving field. Thus a comprehensive understanding to decoherence of a realistic superconducting qubit needs to include influence of driving fields. [18]

In this paper, we study the effect of driving fields on the relaxation and decoherence of a driven qubit. We focus our study on weak resonant driving fields characterized by $\Omega \ll \omega_{\mu}$ (where, $\Omega$ and $\omega_{\mu}$ are the Rabi frequency and the frequency of ac driving field, respectively) and low temperatures. In this case, population leakage to noncomputational states due to strong field effect [54, 55] and thermal activation [56] is negligible and thus a multilevel superconducting qubit coupled to environment and driven by a resonant ac field can be well approximated by a resonantly driven dissipative two-level system (TLS). We first explore relaxation and decoherence of a qubit in free decay and demonstrate that our results agree very well with those in the literature. We then derive analytical expressions of relaxation and decoherence times for a driven qubit through analytical solutions of Bloch equation of the driven dissipative TLS. The relations between the relaxation and decoherence times can be used to extract decoherence and dephasing times of the qubit by measuring its population evolution in free decay and driven oscillation. Finally, we use the analytical expressions to study relaxation and decoherence of a superconducting quantum interface device (SQUID) flux qubit driven by a microwave and show that the analytical results are in excellent agreement with the results obtained by numerically solving the master equation.

\section{BLOCH EQUATION OF A GENERAL DRIVEN DISSIPATIVE TWO-LEVEL SYSTEM}

A dissipative system can be described by a reduced density operator. In the Hilbert space spanned by the eigenstate $|m\rangle$ of eigenenergy $E_{m}(m=1,2, \cdots)$, the reduced density operator $\widehat{\rho}$ is represented by a reduced density matrix with matrix elements $\rho_{m n}=\langle m|\widehat{\rho}| n\rangle$. The diagonal matrix element $\rho_{m m}$ and off-diagonal matrix element $\rho_{m n}(m \neq n)$ are the population and coherence of the system, respectively.

In general, the reduced density operator is governed by generalized master equation of non-Markovian process. [23, 32, 57, 58] However, in the case of weak damping and low temperature considered here, the generalized nonMarkovian master equation is equivalent to the Markovian master equation [32] and in the case of resonant driving, the results obtained from the Markovian master equation are the same as those from the non-Markovian master equation. [57 Furthermore, in the case of weak damping, Lamb shifts are usually very small compared to the ac driving field and thus are often neglected [59]. After dropping the terms related to the Lamb shifts, the Markovian master equation for the reduced density matrix of a driven multilevel qubit is cast into the generalized Bloch-Redfield equation. [56, 60, 61] For a driven dissipative TLS with conserved population, the reduced density matrix elements satisfy $\rho_{21}=\rho_{12}^{*}$ and $\rho_{11}+\rho_{22}=1$ and are governed by [56, 61]

$$
\begin{aligned}
\frac{d \rho_{11}}{d t}= & -i H_{12}^{F}\left(\rho_{21}-\rho_{12}\right)-R_{22,11} \rho_{11} \\
& +R_{11,12}\left(\rho_{12}+\rho_{21}\right)+R_{11,22} \rho_{22} \\
\frac{d \rho_{12}}{d t}= & i \omega_{21} \rho_{12}-i\left[H_{12}^{F}\left(\rho_{22}-\rho_{11}\right)\right. \\
& \left.+\left(H_{11}^{F}-H_{22}^{F}\right) \rho_{12}\right]+R_{12,11} \rho_{11} \\
& +R_{12,12} \rho_{12}+R_{12,21} \rho_{21}+R_{12,22} \rho_{22}
\end{aligned}
$$

where, $\omega_{m n}=\left(E_{m}-E_{n}\right) / \hbar$ is the transition frequency, $R_{m n, m^{\prime} n^{\prime}}$ is the matrix element of damping rate superoperator 
describing the effect of environment on the TLS, [56, 61] and $H_{m n}^{F}=\left\langle m\left|H_{F}\right| n\right\rangle / \hbar$ is the matrix element of interaction Hamiltonian $H_{F}$ between the TLS and resonant ac driving field. For simplicity and without loss of generality, henceforward, unless otherwise specified, we assume $H_{12}^{F}=\varepsilon \cos \left(\omega_{\mu} t\right)$ and $H_{11}^{F}-H_{22}^{F}=\delta \cos \left(\omega_{\mu} t\right)$, where $\varepsilon$ and $\delta$ are the constants associated with transition matrix elements and field strength.

The damping of a dissipative system is caused by the interaction between the system and environment such as a thermal bath. In the case of weak damping, the interaction Hamiltonian $H_{I}$ between the system and the thermal bath can be approximated by a linear function of system variable and expressed as [15, 31, 60] $H_{I}=-\Lambda x \mathcal{U}_{B}$, where, $x$ is the generalized coordinate of system, $\mathcal{U}_{B}$ is a function of bath variable(s), and $\Lambda$ is a coupling constant. The effect of the thermal bath on the system can equivalently be characterized by a spectral density $J(\omega)$. 62, 63] In this case, the matrix elements of the damping rate superoperator can be calculated by [56, 61]

$$
\begin{aligned}
R_{m n, m^{\prime} n^{\prime}}= & \frac{1}{2 \hbar^{2} \Lambda^{2}}\left[-\delta_{n n^{\prime}} \sum_{k} x_{m k} x_{k m^{\prime}} J\left(\omega_{m^{\prime} k}\right)\right. \\
& +x_{m m^{\prime}} x_{n^{\prime} n}\left[J\left(\omega_{n^{\prime} n}\right)+J\left(\omega_{m^{\prime} m}\right)\right] \\
& \left.-\delta_{m m^{\prime}} \sum_{k} x_{n^{\prime} k} x_{k n} J\left(\omega_{n^{\prime} k}\right)\right]
\end{aligned}
$$

where, $x_{m n}$ is the transition matrix element.

An equivalent approach to describe the TLS is to use Bloch vector $(u, v, w)$ defined by

$$
\begin{aligned}
u & =\operatorname{Tr}\left(\rho \sigma_{x}\right)=2 \operatorname{Re} \rho_{12}, \\
v & =\operatorname{Tr}\left(\rho \sigma_{y}\right)=-2 \operatorname{Im} \rho_{12}, \\
w & =\operatorname{Tr}\left(\rho \sigma_{z}\right)=\rho_{11}-\rho_{22} .
\end{aligned}
$$

The components of the Bloch vector $u$ and $v$ represent the real and imaginary parts of the coherence $\rho_{12}$, while the component $w$ is the population difference. Substituting the Bloch vector into the master equation (11) and (2) one obtains the following Bloch equation

$$
\begin{aligned}
\frac{d u}{d t}= & \left(R_{12,12}+R_{12,21}\right) u+\left[\omega_{21}-\delta \cos \left(\omega_{\mu} t\right)\right] v \\
& +\left(R_{12,11}-R_{12,22}\right) w+\left(R_{12,11}+R_{12,22}\right), \\
\frac{d v}{d t}= & -\left[\omega_{21}-\delta \cos \left(\omega_{\mu} t\right)\right] u+\left(R_{12,12}-R_{12,21}\right) v \\
& -2 \varepsilon \cos \left(\omega_{\mu} t\right) w, \\
\frac{d w}{d t}= & 2 R_{11,12} u+2 \varepsilon \cos \left(\omega_{\mu} t\right) v-\left(R_{22,11}+R_{11,22}\right) w \\
& +\left(R_{11,22}-R_{22,11}\right) .
\end{aligned}
$$

\section{RELAXATION AND DECOHERENCE IN THE ABSENCE OF AC DRIVING FIELDS}

Suppose the initial value of the Bloch vector is $\left(u_{0}, v_{0}, w_{0}\right)$. In the absence of ac driving fields (free decay) and under the rotating-wave approximation (RWA), the solution of the Bloch equation is given by (see Appendix A for details)

$$
\begin{aligned}
u & =\left[A \cos \left(\varpi t-\theta_{0}\right)+B \sin (\varpi t)\right] e^{-\kappa t}, \\
v & =\left[-A \sin (\varpi t)+B \cos \left(\varpi t+\theta_{0}\right)\right] e^{-\kappa t}, \\
w & =w_{\infty}+D e^{-\gamma t},
\end{aligned}
$$

where, $\kappa=-R_{12,12}$ is introduced for convenience, $\gamma=R_{22,11}+R_{11,22}$ is the relaxation rate in free decay, $w_{\infty}, \varpi$, and $\theta_{0}$ are the parameters related to the damping rate matrix elements, and $A, B$, and $D$ are the constants determined by the initial condition of the TLS and damping rate matrix elements. These parameters and constants are given by Eqs. (A4) to (A9) in Appendix A. It is shown that the population and coherence undergo simple exponential decays independently at rates $\gamma$ and $\kappa$, respectively, and the coherence components $u$ and $v$ contain fast oscillating factors of frequency $\varpi$. When $t \rightarrow \infty$ the coherence components tend to zero, while the population difference tends to a constant $w_{\infty}$. In the case of zero damping $\left(R_{12,21}=0\right) \varpi=\omega_{21}$ and $\theta_{0}=0$ while in the case of weak damping 
$\left(R_{12,21} \ll \omega_{21}\right) \varpi<\omega_{21}$ and $\theta_{0}>0$. Hence $w_{\infty}$ is the population difference at $t \rightarrow \infty, \varpi$ is the transition frequency modified by the damping, and $\theta_{0}$ is the phase shift due to the damping.

It is shown from Eq. (12) that the population (difference) decays exponentially with the rate of $\gamma$. Hence, the relaxation time is

$$
T_{1}=\gamma^{-1}
$$

From Eqs. (10) and (11) the coherence, $\rho_{12}=(u-i v) / 2 \propto e^{-\kappa t}$, decays exponentially with the rate of $\kappa$. The decoherence time is therefore

$$
T_{2}=\kappa^{-1} .
$$

These results agree very well with those obtained by others. [56] Eqs. [13) and (14) indicate that for free decay $T_{1}$ is independent of $T_{2}$. In general $T_{2}<2 T_{1}$ due to dephasing. [33] The pure dephasing time $T_{\varphi}$ is related to $T_{1}$ and $T_{2}$ by [56]

$$
\frac{1}{T_{\varphi}}=\frac{1}{T_{2}}-\frac{1}{2 T_{1}}
$$

For the general case of a qubit coupled to a thermal bath, using Eq. (3), we obtain

$$
\begin{aligned}
& T_{1}^{-1}=\frac{\left|x_{12}\right|^{2}}{\hbar^{2} \Lambda^{2}}\left[J\left(\omega_{21}\right)+J\left(\omega_{12}\right)\right], \\
& T_{2}^{-1}=\frac{1}{2 T_{1}}+\frac{\left(x_{11}-x_{22}\right)^{2}}{2 \hbar^{2} \Lambda^{2}} J(0),
\end{aligned}
$$

and

$$
T_{\varphi}^{-1}=\frac{\left(x_{11}-x_{22}\right)^{2}}{2 \hbar^{2} \Lambda^{2}} J(0) .
$$

These results demonstrate that the relaxation and dephasing rates of the a qubit are determined by the spectral densities at the transition frequency $\omega=\left|\omega_{21}\right|$ and low frequency $\omega=0$, respectively. [14, 19, 31, 38, 39, [56] In addition, the relaxation rate is proportional to the modulus square of the transition matrix element $\left|x_{12}\right|^{2}$, while the dephasing rate is proportional to the square of the difference of average coordinates of the two states $\left(x_{11}-x_{22}\right)^{2}$. For a qubit having $\left(x_{11}-x_{22}\right)=0$, the dephasing is completely suppressed.

\section{RELAXATION AND DECOHERENCE IN THE PRESENCE OF AN AC DRIVING FIELD}

For a driven dissipative TLS, $\varepsilon \neq 0$ and also $\delta \neq 0$ in general. Under the RWA, the solution of the Bloch equation in the underdamped regime with $\varepsilon>\eta$ is given by (see Appendix B for details)

$$
\begin{aligned}
u= & A \cos \left(\varpi t-\theta_{0}\right) e^{-\kappa t}+B_{0} \sin (\varpi t) \\
& +B_{1} \sin \left(\Omega t+\theta_{1}\right) \sin (\varpi t) e^{-\Gamma t}, \\
v= & -A \sin (\varpi t) e^{-\kappa t}+B_{0} \cos \left(\varpi t+\theta_{0}\right) \\
& +B_{1} \sin \left(\Omega t+\theta_{1}\right) \cos \left(\varpi t+\theta_{0}\right) e^{-\Gamma t}, \\
w= & D_{0}+D_{1} \sin \left(\Omega t+\theta_{2}\right) e^{-\Gamma t},
\end{aligned}
$$

where, $\eta=(\gamma-\kappa) / 2$ is the damping strength, $\Omega=\sqrt{\varepsilon^{2}-\eta^{2}}$ is the Rabi frequency, $\Gamma=(\gamma+\kappa) / 2$ is the mean value of relaxation and decoherence rates in free decay, $A$ is the field-independent constant identical with that in Eqs. (10) and (11) and is given by Eq. (A7) in Appendix A, $B_{0}, B_{1}, D_{0}, D_{1}, \theta_{1}$, and $\theta_{2}$ are the field-dependent constants given by Eqs. (B4) to (B11) in Appendix B. For quantum information processing, manipulation of qubits must be in the underdamped regime. Hence, hereafter our discussion is focused on the resonantly driven qubit in this regime.

It is shown clearly that the solution given by Eqs. (19) to (21) contains a slow Rabi oscillation. It is also shown that in general the population and coherence undergo more complicated damped oscillations which are totally different from those in free decay. The first terms of the coherence components on the right-hand sides of Eqs. (19) and (20) are independent of the driving field. They only depend on the initial state and matrix elements of the damping rate superoperator. Hence they represent intrinsic decoherence induced by the coupling between the qubit and thermal 
bath. Note that the intrinsic decoherence rate $\kappa$ of a qubit in driven oscillation equals to the decoherence rate of free decay (see Eqs. (10) and (11)). In contrast, the remaining terms of the coherence components on the right-hand sides of Eqs. (19) and (20) strongly depend on the driving field. In particular, the third terms decay exponentially and thus represent field-dependent decoherence. The field-dependent decoherence rate $\Gamma$ and the relaxation rate of the qubit in driven oscillation are equal (see Eq. (21)).

Note that both the relaxation and decoherence should have been eliminated in principle if $A=B_{1}=D_{1}=0$ in Eqs. (19) to (21). However, such conditions can not be fulfilled in reality for a resonantly driven qubit starting from a pure state. Thus the relaxation and decoherence can not be suppressed simultaneously with a single resonant driving field. [42, 43, 44, 45, 46, 47] Nevertheless, as will be demonstrated below, for a special class of initial states, a properly chosen resonant driving field could slow down the decoherence. 64]

Since in general the population and coherence of a driven qubit decay with more than one rates, it is necessary to introduce multiple relaxation and decoherence times to describe the system's behavior properly. Each relaxation time or decoherence time represents one of the characteristic times of the qubit for each specific case. For instance, from Eq. (21), the relaxation time $\widetilde{T}_{1}$ is defined by

$$
\widetilde{T}_{1}=\Gamma^{-1}
$$

where the tilde " " is used to indicate that the qubit is resonantly driven. Similarly, from Eqs. (19) and (20), the intrinsic decoherence time $\widetilde{T}_{2,1}$ and field-dependent decoherence time $\widetilde{T}_{2,2}$ are defined by

$$
\widetilde{T}_{2,1}=\kappa^{-1}=T_{2},
$$

and

$$
\widetilde{T}_{2,2}=\Gamma^{-1}=\widetilde{T}_{1},
$$

respectively. Namely, the intrinsic decoherence time $\widetilde{T}_{2,1}$ and the field-dependent decoherence time $\widetilde{T}_{2,2}$ of a qubit in driven oscillation equal to the decoherence time $T_{2}$ of the qubit in free decay and the relaxation time $\widetilde{T}_{1}$ of the qubit in driven oscillation, respectively. If the initial state of the driven qubit corresponds to $u_{0}=0$ (e.g., the ground state), then the intrinsic decoherence terms vanish and the decoherence of the qubit is characterized completely by the field-dependent decoherence time $\widetilde{T}_{2,2}$. Note that the Eqs. (14) and (22) are used to obtain the second equality of Eqs. (23) and (24).

From Eqs. (13) to (15), Eq. (23), and Eq. (24) we obtain

$$
\frac{1}{\widetilde{T}_{2,2}}=\frac{1}{\widetilde{T}_{1}}=\frac{1}{2 T_{1}}+\frac{1}{2 T_{2}}=\frac{3}{4 T_{1}}+\frac{1}{2 T_{\varphi}} .
$$

It indicates that $\widetilde{T}_{2,2}=\widetilde{T}_{1}$ is always in between $T_{1}$ and $T_{2}$ and smaller than the lesser of $4 T_{1} / 3$ and $2 T_{\varphi}$. This prediction is similar to those obtained by others [23, 24, 65] and also agrees, within the experimental uncertainties, with the results of recent experiment. [14]

The relations between various characteristic times are summarized in TABLE I. It is clearly shown that for a driven qubit the intrinsic decoherence time is independent of the driving field and is identical with the decoherence time of the qubit in free decay. In contrast, the relaxation and field-dependent decoherence times of the driven qubit strongly depend on the driving field and are equal to each other.

TABLE I: Relaxation and decoherence times of a qubit in free and driven decays.

\begin{tabular}{cccc}
\hline \hline Decay type & Relaxation & \multicolumn{2}{c}{ Decoherence } \\
\cline { 3 - 4 } & & intrinsic & field-dependent \\
\hline Free decay & $T_{1}=\gamma^{-1}$ & $T_{2}=\kappa^{-1}$ & \\
Driven decay & $\widetilde{T}_{1}=\Gamma^{-1}$ & $\widetilde{T}_{2,1}=T_{2}$ & $\widetilde{T}_{2,2}=\widetilde{T}_{1}$ \\
\hline \hline
\end{tabular}

The relations between the relaxation and decoherence times of a qubit in free decay and driven oscillation can be used to extract the decoherence and dephasing times of the qubit by measuring its population evolution. In experiment, one would first measure the population evolution of the qubit in the free decay and in resonantly driven oscillation (Rabi oscillation) to obtain $T_{1}$ and $\widetilde{T}_{1}$. Then the decoherence times $T_{2}, \widetilde{T}_{2,1}$, and $\widetilde{T}_{2,2}$ can be evaluated using Eqs. (23) to (25). Finally, the pure dephasing time of the qubit $T_{\varphi}$ can be calculated from Eq. (15). 


\section{MICROWAVE-DRIVEN SQUID FLUX QUBITS: ANALYTICAL VERSUS NUMERICAL RESULTS}

To demonstrate validity of the analytical expressions of relaxation and decoherence times obtained in the preceding sections we apply them to calculate relaxation and decoherence times of a microwave-driven SQUID flux qubit inductively coupled to environment and compare the results with those obtained by numerically solving the master equation given by Eqs. (11) and (2). An rf SQUID consists of a superconducting loop of inductance $L$ interrupted by a Josephson tunnel junction (JJ) which, applying the resistively-shunted junction (RSJ) model [66], is characterized by the critical current $I_{c}$, shunt capacitance $C$, and shunt resistance $R$. The SQUID flux qubit is usually coupled to its control and readout circuits. A typical equivalent circuit is shown in FIG. 1 (a) along with its equivalent admittance $Y(\omega)$ in FIG. 1 (b). In this simplified external circuit the left part is the SQUID and the right part supplies external flux to the SQUID qubit. For a superconducting device such as a SQUID, the thermal bath is the external circuit coupled to the device. Thus the external circuit is the dominant source of dissipation for the SQUID qubit [15]. In this section, we analyze the relaxation and decoherence of the SQUID flux qubit due to coupling to the external circuit.

\section{A. Hamiltonian of a microwave-driven SQUID flux qubit}

The Hamiltonian of a flux-biased rf SQUID with total magnetic flux $\Phi$ enclosed in the loop can be written as [22, 67]

$$
H_{0}(x)=\frac{p^{2}}{2 m}+V(x),
$$

where, $m=C \Phi_{0}^{2}$ is the mass of "flux" particle, $\Phi_{0} \equiv h / 2 e$ is the flux quantum, $e$ is the elementary charge, $x=\Phi / \Phi_{0}$ is the canonical coordinate of "flux" particle, $p=-i \hbar \partial / \partial x$ is the canonical momentum conjugate to $x$, and $V(x)$ is the potential energy given by

$$
V(x)=\frac{1}{2} m \omega_{L C}^{2}\left(x-x_{e}\right)^{2}-E_{J} \cos (2 \pi x) .
$$

Here, $E_{J}=\hbar I_{c} / 2 e=m \omega_{L C}^{2} \beta_{L} / 4 \pi^{2}$ is the Josephson coupling energy, $\beta_{L}=2 \pi L I_{c} / \Phi_{0}$ is the potential shape parameter, $\omega_{L C}=1 / \sqrt{L C}$ is the characteristic frequency of the SQUID, and $x_{e}=\Phi_{e} / \Phi_{0}$ is the normalized external fluxes from the external circuit.

To perform gate operations, a microwave pulse is applied to the SQUID qubit. If the interaction between the microwave and external circuit is negligible the Hamiltonian of the microwave-driven SQUID qubit coupled to the external circuit is given by

$$
H(x, t)=H_{0}(x)+H_{F}(x, t)+H_{I}
$$

where, $H_{F}(x, t)$ is the interaction Hamiltonian between the SQUID qubit and microwave and $H_{I}$ is the interaction Hamiltonian between the SQUID qubit and the external circuit (thermal bath).

If $\phi(t)$ is the normalized flux coupled to the SQUID from the microwave then $H_{F}(x, t)$ is given by [67]

$$
H_{F}(x, t)=\frac{m \omega_{L C}^{2}}{2} \phi\left[\phi+2\left(x-x_{e}\right)\right]
$$

Hereafter $\phi$ is taken to be

$$
\phi(t)=\phi_{\mu} \cos \left(\omega_{\mu} t\right)
$$

where, $\phi_{\mu}$ and $\omega_{\mu}$ are the field strength and frequency of the microwave, respectively. From Eqs. (29) and (30) one has

$$
\varepsilon=\frac{m \omega_{L C}^{2} \phi_{\mu}\left|x_{12}\right|}{\hbar},
$$

and

$$
\delta=\frac{m \omega_{L C}^{2} \phi_{\mu}\left(x_{11}-x_{22}\right)}{\hbar} .
$$

For a SQUID qubit, $\delta \neq 0$ if the tunneling distance $\left|x_{11}-x_{22}\right| \neq 0$. 


\section{B. Spectral density of the external circuit}

As has been addressed, in the case of weak damping, the effect of thermal bath on the superconducting system can be described by the spectral density. 62, 63] For the SQUID flux qubit considered here $\Lambda=-1 / \Phi_{0}$ and the spectral density $J(\omega)$ is given by $[61,63$ ]

$$
J(\omega)=\hbar \omega Y_{R}(\omega)\left[1+\operatorname{coth}\left(\frac{\hbar \omega}{2 k_{B} T}\right)\right],
$$

where, $k_{B}$ is the Boltzmann constant, $T$ is the temperature of the thermal bath, and $Y_{R}(\omega)$ is the real part of the frequency-dependent admittance $Y(\omega)$ of the external circuit. [63] For the external circuit of FIG. 1 one has (see Appendix C for details)

$$
Y_{R}(\omega)=\frac{F_{0}(\omega)}{\omega^{2}+G_{0}(\omega)},
$$

where, $F_{0}(\omega)$ and $G_{0}(\omega)$ are given by Eqs. (C8) to 10 in Appendix C. For the sake of concreteness, henceforth, unless otherwise specified, the SQUID flux qubit has the parameters of $L=1.0 \mathrm{nH}, C=15 \mathrm{fF}, L_{e}=1.0 \mathrm{nH}, C_{e}=10$ $\mathrm{pF}, R_{e}=10 \Omega, R_{0}=1.0 \mathrm{k} \Omega, \beta_{L}=1.4, x_{e}=0.499$, and $M=3.0 \mathrm{pH}$. The temperature of the external circuit is taken to be $T=0.1 \mathrm{~K}$.

In FIG. 2 we plot the spectral density $J(\omega)$ versus frequency $\omega$ for the SQUID qubit with the above parameters at $T=0.1 \mathrm{~K}$. It is shown that the spectral density $J(\omega)$ reaches the maximum at $\omega \simeq 0.04 \omega_{L C}$ and approaches a constant value at low frequency, in particular $J(0) \neq 0$. From Eq. (18) $T_{\varphi}$ is finite and thus the external circuit will induce dissipation as well as dephasing.

In FIG. 3, we show the spectral density $J(\omega)$ at $\omega=\omega_{L C}$ versus temperature (the solid curve) together with the quantum spectral density at $T=0$ (the dashed line) and the classical spectral density at high temperature limit $T \gg \hbar \omega_{L C} / k_{B}$ (the dashed dotted line). The quantum spectral density is independent of temperature while the classical spectral density is a linear function of temperature, which agree with those in the literature. 60. Note that for $T>1.5 \mathrm{~K}$ the spectral density is approximated well by the classical spectral density, while $T<0.3 \mathrm{~K}$ the spectral density approaches the quantum spectral density.

For the SQUID flux qubit, $Y_{R}(\omega)$ is an even function of $\omega$, which is also the case for most of superconducting systems. Substituting Eq. (33) into Eqs. (16) and (18), we obtain

$$
T_{1}^{-1}=\frac{2 \pi^{2}}{e^{2}} \hbar \omega_{21}\left|x_{12}\right|^{2} Y_{R}\left(\omega_{21}\right) \operatorname{coth}\left(\frac{\hbar \omega_{21}}{2 k_{B} T}\right)
$$

and

$$
T_{\varphi}^{-1}=\frac{\pi^{2}}{e^{2}} k_{B} T\left(x_{11}-x_{22}\right)^{2} Y_{R}(0)
$$

Thus the relaxation rate is dominated by the circuit's admittance at the transition frequency $\omega_{21}$ while the dephasing rate by the admittance at $\omega=0$. Furthermore, the dephasing rate is proportional to the temperature of thermal bath. Hence at the low temperature the dominating source of decoherence is relaxation while at the high temperature the main source of decoherence is dephasing. These results agree with those obtained by others. [30, 56, 62]

\section{Relaxation and decoherence times}

To numerically calculate the relaxation and decoherence times of the microwave-driven SQUID qubit, we need to compute evolution of population and coherence of the qubit. For this purpose we first calculate the eigenenergy $E_{m}$ and eigenstate $|m\rangle$ of the SQUID qubit by numerically solving the time-independent Schrödinger equation with $H_{0}(x)$. [22, 67] We then calculate the transition frequency $\omega_{m n}$, damping rate matrix element $R_{m n, m^{\prime} n^{\prime}}$, and matrix element of interaction Hamiltonian $H_{m n}^{F}$ between the SQUID qubit and microwave. Finally we calculate the population and coherence by numerically solving the master equation given by Eqs. (11) and (2) using the split-operator method [68] for the algebra equation with non-symmetric matrix. 61] 


\section{Free decay of the SQUID qubit}

In general, a SQUID qubit is a multilevel system. [67] However, when it is driven by a weak resonant microwave field, the leakage to non-computational states is negligible [54, 55] and the SQUID qubit can be very well approximated by a TLS consisting of the lowest two levels $|1\rangle$ and $|2\rangle$ as the computational states.

If the TLS is initially in an eigenstate (the ground or excited state) the coherence of the system will remain zero in free decay. To extract the relaxation and decoherence times of the SQUID qubit from numerically simulated time evolution in free decay we assume the initial state of the qubit is a superposition state with $\rho_{11}(0)=\rho_{12}(0)=$ $\rho_{21}(0)=\rho_{22}(0)=0.5$. From Eqs. (4) to (6), in this case $u_{0}=1$ and $v_{0}=w_{0}=0$. Using this initial state one can calculate population and coherence of the SQUID qubit in free decay by numerically solving the master equation given by Eqs. (11) and (2).

In FIG. 4 (a) and (b) the solid lines are the numerical results of the evolution of population inversion $\left(\rho_{22}-\rho_{11}\right)$ and squared modulus of coherence $\left|\rho_{12}\right|^{2}$, respectively. Since the coherence is usually a complex and fast oscillating quantity, we use $\left|\rho_{12}\right|^{2}$ instead of $\rho_{12}$ to estimate the decoherence time. It is shown that both the population inversion and the squared modulus of coherence undergo simple exponential decays.

To evaluate the relaxation and decoherence times, we fit the numerically obtained $\left(\rho_{22}-\rho_{11}\right)$ and $\left|\rho_{12}\right|^{2}$ with exponential functions

$$
\rho_{22}-\rho_{11}=y_{1}+z_{1} e^{-t / \tau_{1}}
$$

and

$$
\left|\rho_{12}\right|^{2}=y_{2}+z_{2} e^{-2 t / \tau_{2}}
$$

respectively. The results of least-square fitting are plotted in FIG. 4 (a) and (b) with dashed lines, where $\tau_{1}=102.43$ $\mathrm{ns}$ and $\tau_{2}=188.27 \mathrm{~ns}$ (and other fitting parameters are $y_{1}=-0.61198, z_{1}=0.61198, y_{2}=0$, and $z_{2}=0.25$ ). For the weak damping considered here $\theta_{0} \approx 0$. According to Eqs. (10) to (12), for the qubit in free decay, the relaxation time is $T_{1}=\tau_{1}$ and the decoherence time is $T_{2}=\tau_{2}$. Using Eq. (15), the dephasing time $T_{\varphi}=2324.83 \mathrm{~ns}$ for this special case. Note that since in this particular case $T_{\varphi} \gg 2 T_{1}$ the decoherence time is limited by the relaxation time.

For comparison, we calculate the relaxation, decoherence, and dephasing times using the analytical expressions given by Eqs. (16) to (18), Eq. (33), and Eq. (34). The results are $T_{1}=102.43 \mathrm{~ns}, T_{2}=188.27 \mathrm{~ns}$, and $T_{\varphi}=2324.83$ ns. These results are exactly the same as the numerical results, demonstrating the analytical and numerical methods give identical relaxation and decoherence times for the SQUID qubit in free decay within the relative error of the least-square fitting.

\section{Resonantly driven SQUID qubit - Rabi oscillation}

To illustrate the effect of driving field on relaxation and decoherence we consider a SQUID qubit being in the ground state initially driven by a resonant microwave field. For the ground state, $\rho_{11}(0)=1$ and $\rho_{22}(0)=\rho_{21}(0)=\rho_{12}(0)=0$ and thus $u_{0}=v_{0}=0$ and $w_{0}=1$ from Eqs. (4) to (6). In this case, from Eq. (A7) the intrinsic decoherence terms of Eqs. (19) and (20) vanish. With this initial state one can calculate the population and coherence of the microwavedriven SQUID flux qubit by numerically solving the master equation.

In FIG. 5 (a) and (b) we plot with the solid lines the evolution of population difference $\left(\rho_{11}-\rho_{22}\right)$ and squared modulus of coherence $\left|\rho_{12}\right|^{2}$, respectively, for the SQUID flux qubit driven by a microwave with $\phi_{\mu}=1.0 \times 10^{-4}$ and $\omega_{\mu}=\omega_{21}=7.22 \times 10^{-2} \omega_{L C}$, where $\omega_{L C}=2.582 \times 10^{11} \mathrm{rad} / \mathrm{s}$. The damping strength is $\eta=8.62 \times 10^{-6} \omega_{L C}$ for the SQUID qubit considered here while the field strength corresponding to the critically-damped regime is $\phi_{\mu c}=$ $4.94 \times 10^{-7}$ from Eq. (31). Thus the SQUID qubit is in the underdamped regime. As a consequence, both the $\left(\rho_{11}-\rho_{22}\right)$ and $\left|\rho_{12}\right|^{2}$ undergo damped Rabi oscillations with Rabi frequency $\Omega=1.74 \times 10^{-3} \omega_{L C}$, as shown in FIG. 5 (a) and (b).

To extract the relaxation and decoherence times of the driven qubit, we fit the numerically calculated $\left(\rho_{11}-\rho_{22}\right)$ and $\left|\rho_{12}\right|^{2}$ to exponentially damped oscillating functions

$$
\rho_{11}-\rho_{22}=\widetilde{y}_{1}+\widetilde{z}_{1} \sin \left(\Omega t+\varphi_{1}\right) e^{-t / \widetilde{\tau}_{1}},
$$

and

$$
\begin{aligned}
\left|\rho_{12}\right|^{2}= & \widetilde{y}_{2}+\widetilde{z}_{2} \sin \left(\Omega t+\varphi_{2}\right) e^{-t / \widetilde{\tau}_{22}} \\
& +\widetilde{z}_{3} \sin ^{2}\left(\Omega t+\varphi_{2}\right) e^{-2 t / \widetilde{\tau}_{22}}
\end{aligned}
$$


respectively. The results of best fit are shown in FIG. 5 (a) and (b) with dashed lines, where $\widetilde{\tau}_{1}=132.68$ ns and $\widetilde{\tau}_{22}=132.65 \mathrm{~ns}$ (and other fitting parameters are $\widetilde{y}_{1}=0.00033, \widetilde{z}_{1}=0.99976, \varphi_{1}=1.56276, \widetilde{y}_{2}=0.00042$, $\widetilde{z}_{2}=0.00665, \widetilde{z}_{3}=0.24941$, and $\left.\varphi_{2}=-0.01306\right)$. Since $\Omega \ll \omega_{\mu}$ the system is in the weak field regime. According to Eqs. (19) to (21), for the resonantly driven qubit, the relaxation time is $\widetilde{T}_{1}=\widetilde{\tau}_{1}$ and the field-dependent decoherence time is $\widetilde{T}_{2,2}=\widetilde{\tau}_{22}$.

For comparison, we have calculated the relaxation and field-dependent decoherence times using the analytical expressions given by Eq. (25) and Eqs. (16) to (18). The results, $\widetilde{T}_{1}=\widetilde{T}_{2,2}=132.68 \mathrm{~ns}$, are in excellent agreement with the numerical results. We have also calculated the relaxation and decoherence times of the underdamped qubit driven by microwaves of different field strength. The results are given in TABLE II] It is shown that compared to free decay the effect of resonant microwave field is to make the relaxation time longer but the decoherence time shorter. It is also shown that the relaxation and field-dependent decoherence times obtained from the numerical calculation are essentially identical and independent of the field strength, which accord with the analytical results. Note that as the microwave field becomes stronger the numerically obtained decoherence and relaxation times begin to deviate from the analytical results due to strong field effects.

TABLE II: Numerical results of relaxation and decoherence times (ns) of the SQUID qubit in free decay and driven decay.

\begin{tabular}{ccc}
\hline \hline Field strength & Relaxation time & Decoherence time \\
\hline 0 & 102.43 & 188.27 \\
$1 \times 10^{-6}$ & 132.68 & 132.68 \\
$5 \times 10^{-6}$ & 132.68 & 132.68 \\
$1 \times 10^{-5}$ & 132.68 & 132.67 \\
$5 \times 10^{-5}$ & 132.68 & 132.67 \\
$1 \times 10^{-4}$ & 132.68 & 132.65 \\
$2 \times 10^{-4}$ & 132.70 & 132.49 \\
\hline \hline
\end{tabular}

\section{CONCLUSION}

In summary, by analytically solving the Bloch equation of a resonantly driven dissipative TLS under the RWA, the dynamical behavior of a driven qubit is systemically investigated. It is shown that the driving field has significant effect on the relaxation and decoherence of a qubit. For a resonantly driven qubit, the population and coherence undergo more complicated damped oscillations that in general have more than one exponential decay terms. Multiple relaxation and decoherence times are thus required to completely characterize the time evolution of the driven qubit. It is found that the decoherence of a driven qubit can always be decomposed into the intrinsic and field-dependent ones. The intrinsic and field-dependent decoherence times equal to the decoherence time of the qubit in free decay

and relaxation time of the driven qubit, respectively. The relaxation time $\widetilde{T}_{1}$ and field-dependent decoherence time $\widetilde{T}_{2,2}$ of the driven qubit are always in between $T_{1}$ and $T_{2}$ and smaller than the lesser of $4 T_{1} / 3$ and $2 T_{\varphi}$. The analytical expressions for calculation of the relaxation and decoherence times of the driven qubit are derived. These analytical expressions have been used to study relaxation and decoherence of the microwave-driven SQUID qubit. The results are in excellent agreement with those obtained by numerically solving the master equation, confirming the validity of the analytical expressions. The relations between the relaxation and decoherence times of a qubit in free decay and driven damped oscillation are obtained. These relations can be used to extract the decoherence and dephasing times of a qubit by measuring its population evolution with free decay and resonantly driven Rabi oscillation experiments.

\section{Acknowledgments}

This work is supported in part by the NSF (DMR-0325551) and by AFOSR, NSA, and ARDA through DURINT grant (F49620-01-1-0439).

\section{APPENDIX A: THE SOLUTION OF BLOCH EQUATION OF A DISSIPATIVE TLS IN FREE DECAY}

In the absence of ac driving fields (free decay), $\varepsilon=\delta=0$, and the field-dependent terms in the Bloch equitation (7) to (9) vanish. Usually, the coherence components $u$ and $v$ contain fast oscillating factors. Hence the first term on the 
right-hand side of Eq. (9) oscillates rapidly. In the rotating-reference which rotates with the coherence components, the last four terms on the right-hand side of Eq. (7) also oscillate fast. After dropping out all the fast oscillating terms under the RWA, the Bloch equation is approximated to

$$
\begin{aligned}
\frac{d u}{d t} & =\left(R_{12,12}+R_{12,21}\right) u+\omega_{21} v \\
\frac{d v}{d t} & =-\omega_{21} u+\left(R_{12,12}-R_{12,21}\right) v \\
\frac{d w}{d t} & =-\left(R_{22,11}+R_{11,22}\right) w+\left(R_{11,22}-R_{22,11}\right) .
\end{aligned}
$$

Obviously, there is no coupling between the population and coherence in this special case. Because of this the population and coherence evolve independently.

If the initial value of the Bloch vector is $\left(u_{0}, v_{0}, w_{0}\right)$, the solution of Eqs. (A1) to (A3) is then given by Eqs. (10) to (12). The parameters and constants in the solution are given by

$$
\begin{gathered}
w_{\infty}=\frac{R_{11,22}-R_{22,11}}{R_{22,11}+R_{11,22}}, \\
\varpi=\sqrt{\omega_{21}^{2}-R_{12,21}^{2}}, \\
\theta_{0}=2 \tan ^{-1} \sqrt{\frac{\omega_{21}+R_{12,21}}{\omega_{21}-R_{12,21}}}-\frac{\pi}{2}, \\
A=\frac{u_{0}}{\cos \theta_{0}}, \\
B=\frac{v_{0}}{\cos \theta_{0}},
\end{gathered}
$$

and

$$
D=w_{0}-w_{\infty}
$$

\section{APPENDIX B: SOLUTIONS OF BLOCH EQUATION OF A RESONANTLY DRIVEN DISSIPATIVE TLS}

For a driven dissipative TLS, $\varepsilon \neq 0$ and also $\delta \neq 0$ in general, as will be shown in section V. In this case, we assume that the trial solution of the Bloch equation (77) to (9) still has the form of Eqs. (10) to (12) but with the constants $A, B$, and $D$ replaced by time-dependent variables $\mu, \nu$, and $\lambda$. Substituting the trial solution into Eqs. (7) to (9) we obtain three equations with respect to the variables $\mu, \nu$, and $\lambda$. In the case of the resonant driving field with $\omega_{\mu}=\varpi$, using the RWA, these equations are simplified to the form

$$
\begin{aligned}
& \frac{d \mu}{d t}=0, \\
& \frac{d \nu}{d t}=-\frac{\varepsilon}{\cos \left(\theta_{0}\right)}\left(\lambda e^{-2 \eta t}+w_{\infty} e^{\kappa t}\right), \\
& \frac{d \lambda}{d t}=\nu \varepsilon \cos \left(\theta_{0}\right) e^{2 \eta t}
\end{aligned}
$$

where, $\eta=(\gamma-\kappa) / 2$ is the damping strength. Eqs. (B2) and (B3) indicate that due to the driving field the population and coherence are coupled to each other. 64] Due to this coupling they no longer evolve independently.

The form of the solution of Eqs. (B1) to (B3) depends on the relative value of $\varepsilon$ to $\eta$. According to this relative value the dynamics of the driven dissipative TLS can be categorized into three regimes: underdamped regime when $\varepsilon>\eta$, critically-damped regime when $\varepsilon=\eta$, and overdamped regime when $\varepsilon<\eta$. For the underdamped regime with 
$\varepsilon>\eta$, the most important scheme for quantum computation, the solution of Bloch equation is given by Eqs. (19) to (21). The parameters and constants in the solution are given by

$$
\begin{gathered}
B_{0}=-\frac{\chi \varepsilon}{\cos \theta_{0}}, \\
B_{1}=\frac{\left|w_{0}-\kappa \chi\right|}{\cos \left(\theta_{0}\right)} \sqrt{1+\frac{1}{\Omega^{2}}\left(\eta-\varepsilon \frac{v_{0}+\varepsilon \chi}{w_{0}-\kappa \chi}\right)^{2}}, \\
D_{0}=\kappa \chi \\
D_{1}=B_{1} \cos \theta_{0}, \\
\theta_{1}=\theta_{2}+\theta_{3}, \\
\theta_{2}=\tan ^{-1} \frac{\left.w_{0}-\kappa \chi\right)}{\varepsilon v_{0}-\eta w_{0}+\left(\eta \kappa+\varepsilon^{2}\right) \chi} \\
\theta_{3}=\tan ^{-1} \frac{\Omega}{\eta},
\end{gathered}
$$

and

$$
\chi=\frac{\gamma w_{\infty}}{\varepsilon^{2}+\kappa \gamma}
$$

For the overdamped regime with $\varepsilon<\eta$, the solution of Bloch equation can be obtained by replacing $\Omega$ with $i \sqrt{\eta^{2}-\varepsilon^{2}}$ in Eqs. (19) to (21). In this case, the population difference and coherence components evolve with more than one exponential decaying terms and the coherence components are composed of intrinsic and field-dependent decoherences. For the critically-damped regime with $\varepsilon=\eta$, the solution of Bloch equation can be obtained by setting $\Omega=0$ in Eqs. (19) to (21). In this case, the system undergoes a nonexponential decay owing to the nonexponential decay factor $t e^{-\Gamma t}$ in the population difference and coherence components. Note that the analytical solutions obtained here have been confirmed by the matrix exponent method. [69]

\section{APPENDIX C: EQUIVALENT ADMITTANCE OF THE EXTERNAL CIRCUIT}

In this paper we assume that the circuit denoted by $Y_{e}(\omega)$ in FIG. 1 (a) is an $R C$ circuit with effective admittance $Y_{e}(\omega)$ given by

$$
Y_{e}(\omega)=\frac{1}{R_{0}}+\frac{1}{R_{e}+1 / j \omega C_{e}} .
$$

The corresponding impedance $Z_{e}$ is given by

$$
Z_{e}(\omega)=\frac{1}{Y_{e}(\omega)}=R_{e f f}(\omega)+\frac{1}{j \omega C_{e f f}(\omega)},
$$

where, $R_{\text {eff }}$ and $C_{\text {eff }}$ are the effective resistance and capacity given by

$$
R_{e f f}(\omega)=\frac{R_{0}+\omega^{2} C_{e}^{2} R_{e} R_{0}\left(R_{e}+R_{0}\right)}{1+\omega^{2} C_{e}^{2}\left(R_{e}+R_{0}\right)^{2}},
$$

and

$$
C_{e f f}(\omega)=C_{e}\left[\frac{1}{\omega^{2} R_{0}^{2} C_{e}^{2}}+\left(\frac{R_{e}+R_{0}}{R_{0}}\right)^{2}\right]
$$


respectively. They are functions of $\omega$. When $R_{0} \rightarrow \infty, R_{e f f}=R_{e}$ and $C_{e f f}=C_{e}$.

For the external circuit shown in FIG. 1 (a), the circuit equations are

$$
\begin{aligned}
V_{1} & =j \omega L I_{1}-j \omega M I_{2}, \\
0 & =-j \omega M I_{1}+\left(j \omega L_{e}+R_{e f f}+\frac{1}{j \omega C_{e f f}}\right) I_{2} .
\end{aligned}
$$

The equivalent impedance $Z(\omega)$ is therefore calculated from

$$
Z(\omega)=\frac{V_{1}}{I_{1}}=j \omega L+\frac{\omega^{2} M^{2}}{j \omega L_{e}+R_{e f f}+1 / j \omega C_{e f f}} .
$$

The equivalent admittance $Y(\omega)$ can be calculated from the equivalent impedance $Z(\omega)$ by $Y(\omega)=1 / Z(\omega)$. The real part of the equivalent admittance, $Y_{R}(\omega)$, is given by Eq. (34), where,

$$
\begin{gathered}
F_{0}(\omega)=\frac{M^{2} R_{e f f}}{\varsigma^{2}}, \\
G_{0}(\omega)=\frac{2 L}{\varsigma C_{e f f}}+\frac{L^{2}}{\varsigma^{2}}\left(R_{e f f}^{2}+\frac{1}{\omega^{2} C_{e f f}^{2}}\right),
\end{gathered}
$$

and

$$
\varsigma=M^{2}-L L_{e} .
$$

Eq. (34) shows that $Y_{R}(\omega)$ is an even function of $\omega$. It is also the case for most of superconducting qubits.

[1] Y. Nakamura, Yu. A. Pashkin, and J. S. Tsai, Nature (London) 398, 786 (1999).

[2] D. Vion, A. Aassime, A. Cottet, P. Joyez, H. Pothier, C. Urbina, D. Esteve, and M. H. Devoret, Science 296, 886 (2002).

[3] Y. Yu, S. Han, X. Chu, S.-I. Chu, and Z. Wang, Science 296, 889 (2002).

[4] J. M. Martinis, S. Nam, J. Aumentado, and C. Urbina, Phys. Rev. Lett. 89, 117901 (2002).

[5] J. R. Friedman, V. Patel, W. Chen, S. K. Tolpygo, and J. E. Lukens, Nature (London) 406, 43 (2000).

[6] C. H. van der Wal, A. C. J. ter Haar, F. K. Wilhelm, R. N. Schouten, C. J. P. M. Harmans, T. P. Orlando, S. Lloyd, and J. E. Mooij, Science 290, 773 (2000).

[7] I. Chiorescu, Y. Nakamura, C. J. P. M. Harmans, and J. E. Mooij, Science 299, 1869 (2003).

[8] I. Chiorescu, P. Bertet, K. Semba, Y. Nakamura, C. J. P. M. Harmans, and J. E. Mooij, Nature 431, 159 (2004).

[9] Yu. A. Pashkin, T. Yamamoto, O. Astafiev, Y. Nakamura, D. V. Averin, and J. S. Tsai, Nature (London) 421, 823 (2003).

[10] T. Yamamoto, Yu. A. Pashkin, O. Astafiev, Y. Nakamura, and J. S. Tsai, Nature (London) 425, 941 (2003).

[11] A. J. Berkley, H. Xu, R. C. Ramos, M. A. Gubrud, F. W. Strauch, P. R. Johnson, J. R. Anderson, A. J. Dragt, C. J. Lobb, and F. C. Wellstood, Science 300, 1548 (2003).

[12] R. McDermott, R. W. Simmonds, M. Steffen, K. B. Cooper, K. Cicak, K. D. Osborn, S. Oh, D. P. Pappas, and J. M. Martinis, Science 307, 1299 (2005).

[13] O. Astafiev, Yu. A. Pashkin, Y. Nakamura, T. Yamamoto, and J. S. Tsai, Phys. Rev. Lett. 93, 267007 (2004).

[14] G. Ithier, E. Collin, P. Joyez, P. J. Meeson, D. Vion, D. Esteve, F. Chiarello, A. Shnirman, Y. Makhlin, J. Schriefl, and G. Schön, Phys. Rev. B 72, 134519 (2005).

[15] Y. Makhlin, G. Schon, and A. Shnirman, Rev. Mod. Phys. 73, 357 (2001).

[16] J. E. Mooij, T. P. Orlando, L. Levitov, L. Tian, C. H. van der Wal, and S. Lloyd, Science 285, 1036 (1999).

[17] G. Burkard, D. P. DiVincenzo, P. Bertet, I. Chiorescu, and J. E. Mooij, Phys. Rev. B 71, 134504 (2005).

[18] M. C. Goorden, M. Thorwart, and M. Grifoni, Phys. Rev. Lett. 93, 267005 (2004).

[19] Y. Makhlin and A. Shnirman, Phys. Rev. Lett. 92, 178301 (2004).

[20] T. P. Orlando, L. Tian, D. S. Crankshaw, S. Lloyd, C. H. van der Wal, J. Mooij, and F. Wilhelm, Physica C 368, 294 (2002).

[21] D. J. Van Harlingen, T. L. Robertson, B. L. T. Plourde, P. A. Reichardt, T. A. Crane, and J. Clarke, Phys. Rev. Rev. B 70, 064517 (2004).

[22] Z. Zhou, S.-I. Chu, and S. Han, Phys. Rev. B 70, 094513 (2004).

[23] S. Shresta, C. Anastopoulos, A. Dragulescu, and B. L. Hu, Phys. Rev. A 71, 022109 (2005).

[24] C. Anastopoulos and B. L. Hu, Phys. Rev. A 62, 033821 (2000). 
[25] T. L. Robertson, B. L. T. Plourde, T. Hime, S. Linzen, P. A. Reichardt, F. K. Wilhelm, and J. Clarke, Phys. Rev. B 72, 024513 (2005).

[26] Y. C. Cheng and R. J. Silbey, Phys. Rev. A 69, 052325 (2004).

[27] M. J. Storcz and F. K. Wilhelm, Phys. Rev. A 67, 042319 (2003).

[28] M. Thorwart and P. Hänggi, Phys. Rev. A 65, 012309 (2001).

[29] M. Governale, M. Grifoni, and G. Schn, Chem. Phys. 268, 273 (2001).

[30] L. Tian, S. Lloyd, and T. P. Orlando, Phys. Rev. B 65, 144516 (2002).

[31] H. Xu, A. J. Berkley, R. C. Ramos, M. A. Gubrud, P. R. Johnson, F. W. Strauch, A. J. Dragt, J. R. Anderson, C. J. Lobb, and F. C. Wellstood, Phys. Rev. B 71, 064512 (2005).

[32] L. Hartmann, I. Goychuk, M. Grifoni, and P. Hänggi, Phys. Rev. E 61, R4687 (2000).

[33] G. Falci, A. D'Arrigo, A. Mastellone, and E. Paladino, Phys. Rev. Lett. 94, 167002 (2005).

[34] Y. Nakamura, Yu. A. Pashkin, T. Yamamoto, and J. S. Tsai, Phys. Rev. Lett. 88, 047901 (2002).

[35] K. W. Lehnert, K. Bladh, L. F. Spietz, D. Gunnarson, D. I. Schuster, P. Delsing, and R. J. Schoelkopf, Phys. Rev. Lett. 90, 027002 (2003).

[36] T. Duty, D. Gunnarsson, K. Bladh, and P. Delsing, Phys. Rev. B 69, 140503(R) (2004).

[37] S.-X. Li, W. Qiu, Z. Zhou, M. Matheny, W. Chen, J. E. Lukens, and S. Han, arXiv: cond-mat/0507008 (2005).

[38] P. Bertet, I. Chiorescu, G. Burkard, K. Semba, C. J. P. M. Harmans, D. DiVincenzo, and J. E. Mooij, arXiv:condmat/0412485 (unpublished).

[39] A. J. Berkley, H. Xu, M. A. Gubrud, R. C. Ramos, J. R. Anderson, C. J. Lobb, and F. C. Wellstood, Phys. Rev. B 68, 060502(R) (2003).

[40] S. K. Dutta, H. Xu, A. J. Berkley, R. C. Ramos, M. A. Gubrud, J. R. Anderson, C. J. Lobb, and F. C. Wellstood, Phys. Rev. B 70, 140502(R) (2004).

[41] L. Viola and E. Knill, Phys. Rev. Lett. 94, 060502 (2005).

[42] L. Viola and S. Lloyd, Phys. Rev. A 58, 2733 (1998).

[43] L. Viola, E. Knill, and S. Lloyd, Phys. Rev. Lett. 82, 2417 (1999).

[44] D. Vitali and P. Tombesi, Phys. Rev. A 65, 012305 (2002).

[45] H. Gutmann, F. K. Wilhelm, W. M. Kaminsky, and S. Lloyd, Phys. Rev. A 71, 020302(R) (2005).

[46] G. Falci, A. D'Arrigo, A. Mastellone, and E. Paladino, Phys. Rev. A 70, 040101(R) (2004).

[47] L. Faoro and L. Viola, Phys. Rev. Lett. 92, 117905 (2004).

[48] K. Shiokawa and D. A. Lidar, Phys. Rev. A 69, 030302(R) (2004).

[49] L.-M. Duan and G.-C. Guo, Phys. Rev. Lett. 79, 1953 (1997).

[50] P. Zanardi and M. Rasetti, Phys. Rev. Lett. 79, 3306 (1997).

[51] D. A. Lidar, I. L. Chuang, and K. B. Whaley, Phys. Rev. Lett. 81, 2594 (1998).

[52] A. Beige, D. Braun, B. Tregenna, and P. L. Knight, Phys. Rev. Lett. 85, 1762 (2000).

[53] D. Bacon, K. R. Brown, and K. B. Whaley, Phys. Rev. Lett. 87, 247902 (2001).

[54] Z. Zhou, S.-I. Chu, and S. Han, Phys. Rev. Lett. 95, 120501 (2005).

[55] Z. Zhou, S.-I. Chu, and S. Han, Phys. Rev. B 73, 104521 (2006).

[56] G. Burkard, R. H. Koch, and D. P. DiVincenzo, Phys. Rev. B 69, 064503 (2004).

[57] J. R. Brinati, S. S. Mizrahi, and G. A. Prataviera, Phys. Rev. A 50, 3304 (1994).

[58] A. Yu. Smirnov, Phys. Rev. B 67, 155104 (2003).

[59] W. H. Louisell, Quantum Statistical Properties of Radiation (John Wiley \& Sons, 1973).

[60] U. Weiss, Quantum Dissipative Systems (World Scientific Publishing Co., Singapore, 1999), 2nd ed.

[61] Z. Zhou, S.-I. Chu, and S. Han, Phys. Rev. B (to be submitted).

[62] A. Leggett, S. Chakravarty, A. Dorsey, M. Fisher, A. Garg, and W. Zwerger, Rev. Mod. Phys. 59, 1 (1987).

[63] M. H. Devoret, in Quantum Fluctuations, edited by S. Reynaud, E. Giacobino, and J. Zinn-Justin, Les Houches, France, 27 June-28 July 1995 (Elsevier Science B. V., 1997), pp. 351-386.

[64] M. Grifoni, E. Paladino, and U.Weiss, Eur. J. Phys. B 10, 719 (1999).

[65] N. Kosugi, S. Matsuo, K. Konno, and N. Hatakenaka, Phys. Rev. B 72, 172509 (2005).

[66] V. V. Danilov, K. Likharev, and A. B. Zorin, IEEE Trans. Magn. 19, 572 (1983).

[67] Z. Zhou, S.-I. Chu, and S. Han, Phys. Rev. B 66, 054527 (2002).

[68] M. R. Hermann and J. A. Fleck, Jr., Phys. Rev. A 38, 6000 (1988).

[69] A. V. Alekseev and N. V. Sushilov, Phys. Rev. A 46, 351 (1992). 


\section{Figure Captions}

FIG. 1 (a) A simplified external circuit of the SQUID flux qubit. The left part is the SQUID qubit and the right part is the circuit used to supply external flux to the SQUID qubit. $I_{1}$ and $V_{1}$ are the current and voltage of the SQUID qubit's circuit, $I_{2}$ is the current of the external circuit, $L_{e}$ is the inductance of the superconducting loop of the external circuit, $M$ is the mutual inductance of the SQUID qubit and external circuit, and $Y_{e}(\omega)$ is the effective admittance of other devices in the external circuit. (b) The equivalent admittance $Y(\omega)$ of the external circuit.

FIG. 2 (Online color) $J(\omega)$ vs. $\omega$ for the SQUID flux qubit at $T=0.1 \mathrm{~K}$.

FIG. 3 (Online color) $J(\omega)$ vs. $T$ for the SQUID flux qubit at $\omega=\omega_{L C}$. The dashed and dashed dotted lines are the spectral densities at low temperature limit $(T \rightarrow 0)$ and high temperature limit $(T \rightarrow \infty)$, respectively.

FIG. 4 (Online color) Evolution of (a) the population inversion and (b) the absolutely squared coherence of the SQUID flux qubit in free decay. The solid and dashed lines are the numerical and fitting results, respectively.

FIG. 5 (Online color) Evolution of (a) the population difference and (b) the absolutely squared coherence of the microwave-driven SQUID flux qubit. The solid and dashed lines are the numerical and fitting results, respectively. 


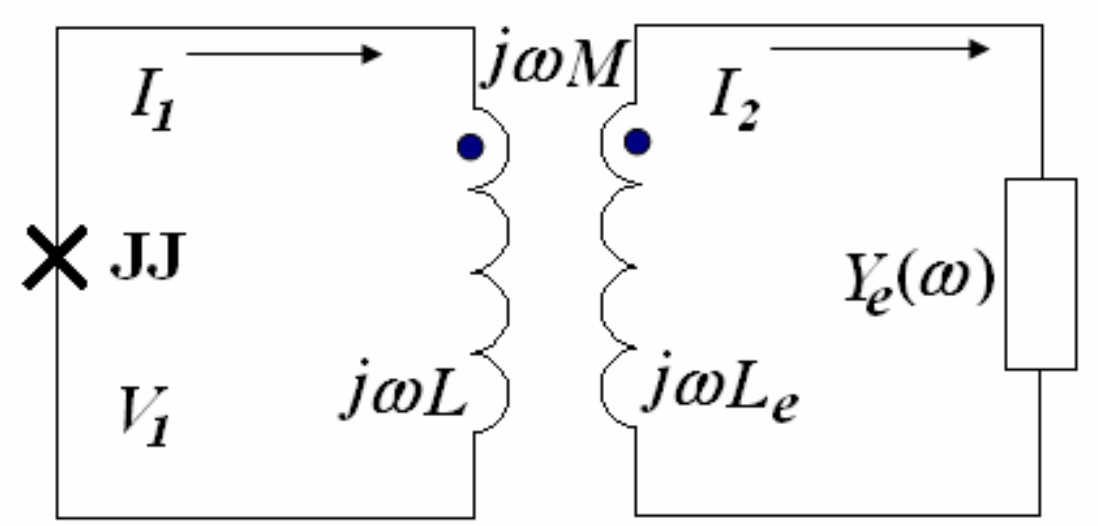

(a)

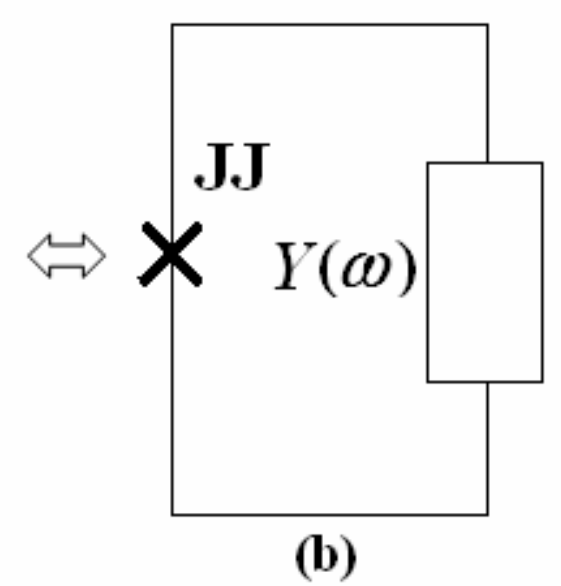

FIG. 1 


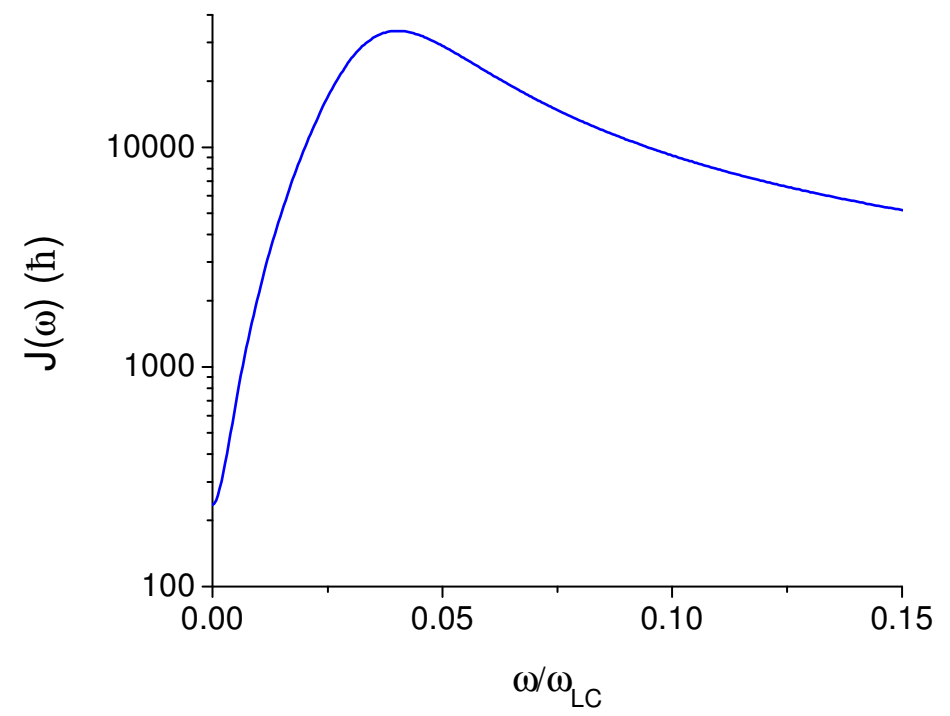

FIG. 2 


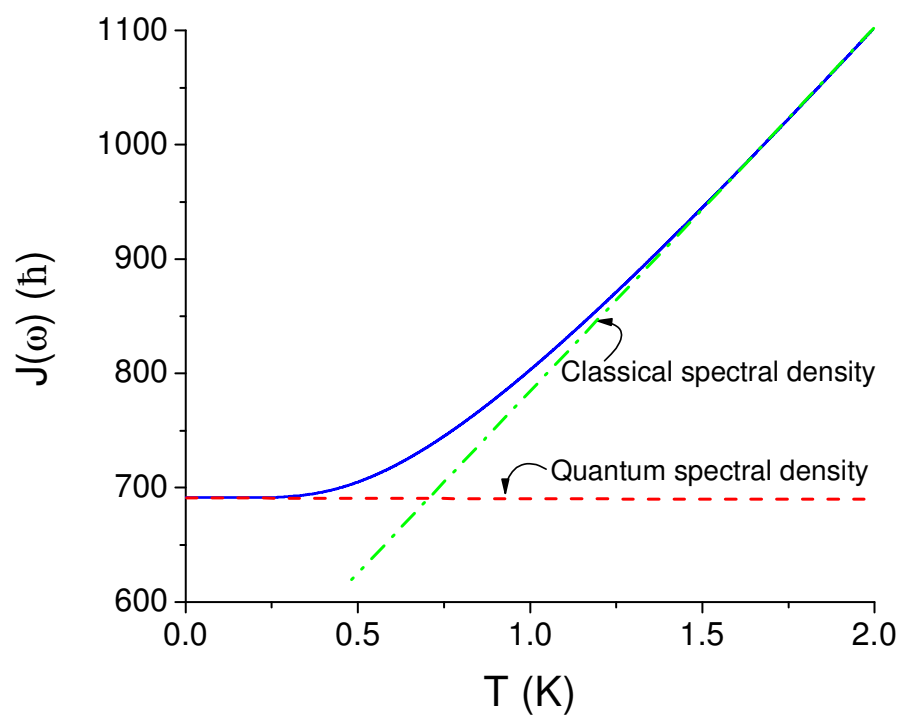

FIG. 3 

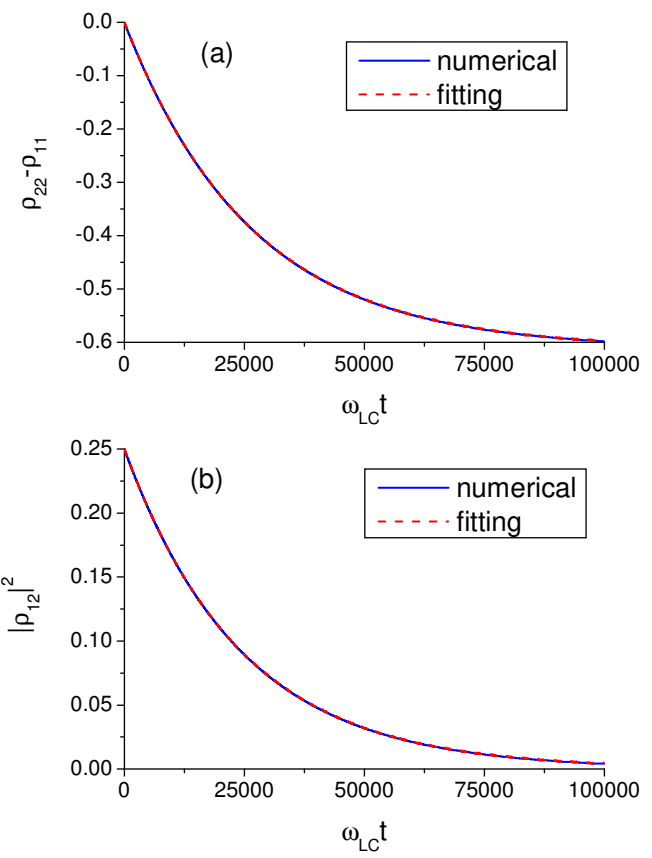

FIG. 4 

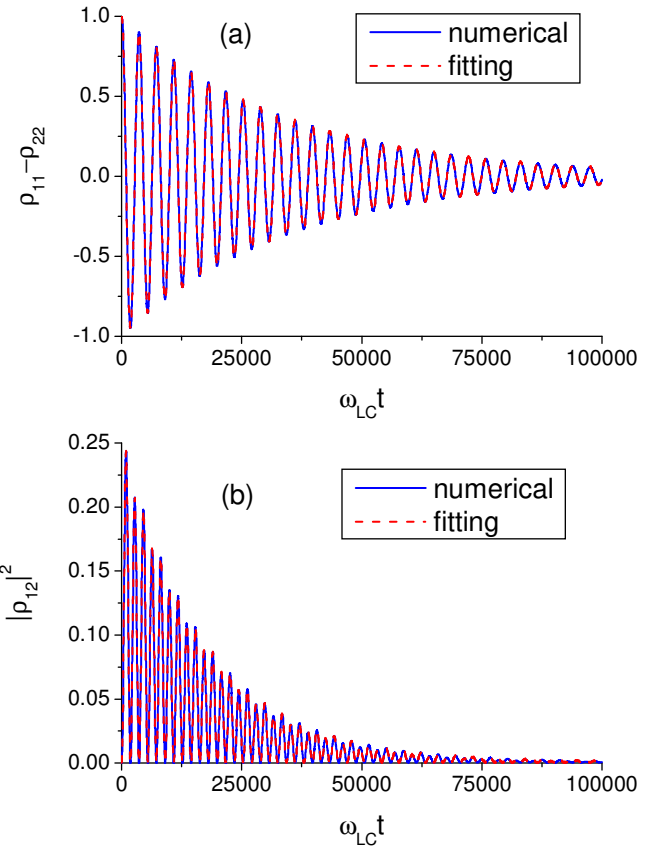

FIG. 5 